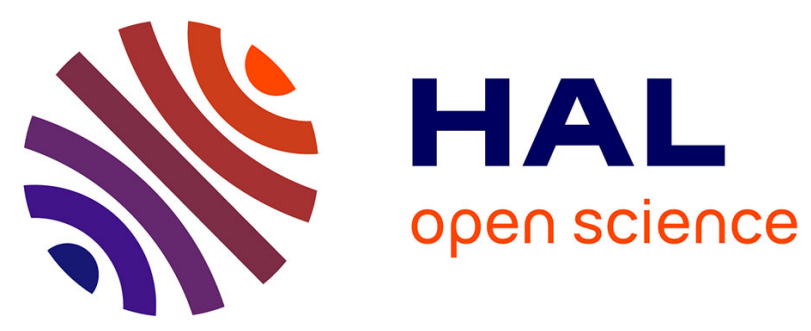

\title{
Computational Performance of Time-Resolved Diffuse Optical Tomography in a Two-Layer Brain Model
}

\author{
David Orive-Miguel, Lionel Hervé, Jerome I. Mars, Laurent Condat,
} Jean-Marc Dinten, Sophie Morales

\section{- To cite this version:}

David Orive-Miguel, Lionel Hervé, Jerome I. Mars, Laurent Condat, Jean-Marc Dinten, et al.. Computational Performance of Time-Resolved Diffuse Optical Tomography in a Two-Layer Brain Model. OSA Biophotonics Congress: Biomedical Optics, Apr 2018, Hollywood, Florida, United States. hal01965639

\section{HAL Id: hal-01965639 \\ https://hal.science/hal-01965639}

Submitted on 26 Dec 2018

HAL is a multi-disciplinary open access archive for the deposit and dissemination of scientific research documents, whether they are published or not. The documents may come from teaching and research institutions in France or abroad, or from public or private research centers.
L'archive ouverte pluridisciplinaire HAL, est destinée au dépôt et à la diffusion de documents scientifiques de niveau recherche, publiés ou non, émanant des établissements d'enseignement et de recherche français ou étrangers, des laboratoires publics ou privés. 


\title{
Computational Performance of Time-Resolved Diffuse Optical Tomography in a Two-Layer Brain Model
}

\author{
David Orive-Miguel $^{1,2}$, Lionel Hervé ${ }^{1}$, Jérôme Mars ${ }^{2}$, Laurent Condat ${ }^{2}$, \\ Jean-Marc Dinten ${ }^{1}$ and Sophie Morales ${ }^{1}$ \\ 1: CEA, LETI, MINATEC Campus, F-38054 Grenoble, France \\ 2: Univ. Grenoble Alpes, CNRS, Grenoble INP, GIPSA-lab, 38000 Grenoble, France \\ david.orive-miguel@cea.fr
}

\begin{abstract}
We evaluate Mellin-Laplace algorithm in a two-layer brain model with MonteCarlo generated data. Results show accurate absorption estimation and the flexibility of the algorithm to detect each layer thickness.
\end{abstract}

OCIS codes: $170.6920,110.6955,110.0113,030.5260,100.3190$

\section{Introduction}

Traumatic brain injury (TBI) is one of the leading causes of disability and injury-related deaths. Although magnetic resonance imaging or computerized x-ray tomography are standard techniques in TBI treatment protocols there is still a need of a technology that could allow to perform bedside, continuous and non-invasive brain monitoring. Timeresolved diffuse optical tomography (TR-DOT) is an emerging photonics technology that could potentially permit to continuously measure important brain physiological values in TBI cases such as blood oxygenation levels.

Even though TR-DOT has already been applied, for example, to flap viability assessment [1] with very promising results there is still a lack of accurate optical properties estimation for brain imaging cases. Nonlinear Monte-Carlo fitting methods have been used in computational analysis [2] but their performance depends significantly on the knowledge of the geometry being scanned and is time consuming. In this work we computationally evaluate an algorithm based on Mellin-Laplace moments and we analyze its accuracy and adaptation to different layer thicknesses.

\section{Method}

The computational phantom consists of a two-layer model $\left(8 \times 8 \times 5 \mathrm{~cm}^{3}\right)$ containing an extracerebral and cerebral layer with one light source and four detectors placed at $1 \mathrm{~cm}$ distance at the top boundary, see Figure 1a. Monte-Carlo simulations [3] were performed on the phantom, signals were convolved with the instrumental response function (IRF) of single-photon avalanche diode (SPAD) and silicon photomultipliers (SiPM) detectors, and were corrupted with Poisson noise.

For the reconstruction of the optical properties, Mellin-Laplace moments algorithm [4] with twelve moments and $p=9$ was used. This algorithm is based on finite element method and computes the moments of time-point spread functions in order to achieve a good balance between accuracy and computational time. The algorithm can be used on experimental data by performing measurements in a reference phantom; moreover, knowledge of the shape of the IRF is not necessary.

\section{Results and Discussion}

The first experiment consists in evaluating the Mellin-Laplace algorithm performance for estimating the absorption at cerebral layer. Monte-Carlo simulations were done with a constant absorption of $\mu_{a, 1}=0.1 \mathrm{~cm}^{-1}$ at the extracerebral layer and an increasing absorption from $\mu_{a, 2}=0.1$ to $0.5 \mathrm{~cm}^{-1}$ on steps of $0.05 \mathrm{~cm}^{-1}$ for the cerebral layer. The scattering coefficient was maintained homogeneous along both layers because of its little sensitivity at brain layer [2]. Extracerebral and cerebral layer thicknesses were $1 \mathrm{~cm}$ and $4 \mathrm{~cm}$ respectively; homogeneous absorption through each layer was assumed. An homogeneous phantom of $\mu_{a}=0.1 \mathrm{~cm}^{-1}$ was taken as reference measurement. Results are plotted in Figure 1b for different types of detectors; they show that Mellin-Laplace algorithm reconstructs linear absorption estimations with low relative error. It is also seen that IRFs influence the results by yielding an overestimation 


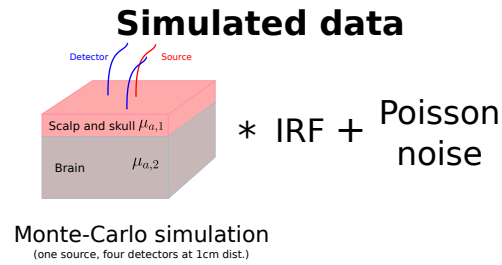

(a) Process for creating simulated data that mimics experimental data.

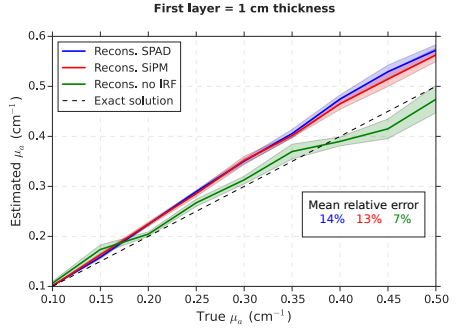

(b) Absorption reconstruction for simulated data with and without IRF. Confidence interval of $75 \%$.

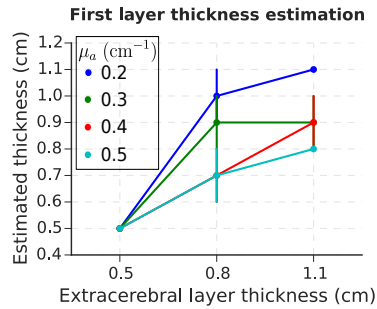

(c) Layer thickness estimation without using a priori data. Confidence interval of $75 \%$.

Fig. 1: Results using Mellin-Laplace moments algorithm.

at absorption values much higher than the reference measurement making the relative error to increase twice with respect to free IRF data. No significant differences are seen in the performance with SPAD or SiPM detectors.

In the second experiment we aim to evaluate whether the algorithm is able to detect the thickness of the two layers; that is, whether the algorithm has the flexibility to adapt to the two-layer geometry when thickness of each layer is not given in advance. The domain was segmented in $0.3 \mathrm{~cm}$-thick layers each of them with an homogeneous absorption. The two-layer geometry interface was estimated as the layer interface with largest absorption difference between above and below layers. Monte-Carlo simulations were performed for cases were extracerebral layer thickness ranged from 0.5 to $1.1 \mathrm{~cm}$ on steps of $0.3 \mathrm{~cm}$; cerebral layer absorption ranged from $\mu_{a, 2}=0.2$ to $0.5 \mathrm{~cm}^{-1}$ on steps of $0.1 \mathrm{~cm}^{-1}$. Results in Figure 1c show that accurate extracerebral layer thickness is estimated in 0.5 and $0.8 \mathrm{~cm}$ cases. Nevertheless, thickness is underestimated in $1.1 \mathrm{~cm}$ case because the algorithm has less sensibility to deeper layers. Higher absorption values tend to underestimate the thickness because the algorithm tries to compensate the high absorption by decreasing the extracerebral layer thickness.

\section{Conclusions and Future Work}

We studied the performances of a Mellin-Laplace algorithm for two-layer brain models with data that mimics realistic conditions. Accurate results are obtained if each layer thickness is given in advance although the algorithm is also capable of doing good estimations without that a priori information.

In the near future, we will extend the analysis by performing Monte-Carlo simulations on ATLAS head models and doing reconstructions of the absorption at the brain layer.

\section{Acknowledgements}

This project has received funding from the European Union's Horizon 2020 Marie Skodowska-Curie Innovative Training Networks (ITN-ETN) programme, under grant agreement no 675332 BitMap. The authors thank to the photonics lab at Politecnico de Milano for providing the instrumental response functions of the detectors.

\section{References}

1. L. Di Sieno, G. Bettega, M. Berger, C. Hamou, M. Aribert, A. Dalla Mora, A. Puszka, H. Grateau, D. Contini, L. Hervé, J.-L. Coll, J.-M. Dinten, A. Pifferi, A. Planat-Chrétien, "Time-resolved diffuse optical tomography for non-invasive flap viability assessment: pre-clinical tests on rats" in European Conferences on Biomedical Optics 2015 Proceedings Volume 9538, Diffuse Optical Imaging V; 95380N (2015).

2. J. Selb, T.M. Ogden, J. Dubb, Q. Fang, D.A. Boas, "Comparison of a layered slab and an atlas head model for Monte Carlo fitting of time-domain near-infrared spectroscopy data of the adult head" in J. Biomed. Opt 2014 Jan; 19(1): 01601.

3. Q. Fang, "Mesh-based Monte Carlo method using fast ray-tracing in Plcker coordinates" in Biomed. Opt. Express Vol. 1, Issue 1, pp. 165-175 (2010).

4. J. Zouaoui, L. Di Sieno, L. Hervé, A. Pifferi, A. Farina, A. Dalla Mora, J. Derouard, J.-M. Dinten, "Quantification in time-domain diffuse optical tomography using Mellin-Laplace transforms" in Biomed. Opt. Express Vol. 7, Issue 10, pp. 4346-4363 (2016). 